\title{
Rational Integration of Photovoltaics for Solar Hydrogen Generation
}

\author{
Dr Fiona J Beck*, \\ Research School of Electrical, Energy and Materials Engineering, Australian National University, lan Ross \\ Building, 31 North Road, Acton, ACT, 2601, Australia \\ *fiona.beck@anu.edu.au
}

\begin{abstract}
The development of commercially viable solar hydrogen generators needs to be accelerated to meet the needs of the emerging global hydrogen economy. Many different hydrogen generators have been demonstrated on a lab-scale, employing semiconductor photovoltaic components integrated into the system in a variety of ways: as solar cells, photoelectrodes and photoelectrochemical cells. Despite this the effect of system configuration on performance has not yet been independently considered. In this work, we demonstrate that the way in which the photovoltaic components are integrated into the system is critical to maximise the solar-tohydrogen conversion efficiency. We introduce a new framework, based on simple equivalent circuit models, to show that decoupling the PV components from the electrochemical cell by employing power management can significantly increase the system efficiency. Decoupled systems can also take advantage of existing solar cell technologies, and the maturity of the silicon PV industry, to rapidly advance solar hydrogen generation.
\end{abstract}

Key Words: hydrogen, solar fuels, photovoltaics, solar energy, photoelectrochemistry, photoelectrodes, tandem solar cells

\section{INTRODUCTION}

The 2018 IPCC report issued a stark warning: $\mathrm{CO}_{2}$ emissions must be reduced to $45 \%$ of their 2010 level by 2030 to limit global warming to $1.5^{\circ} \mathrm{C}$ and avoid the worst effects of climate change ${ }^{1}$. The International Renewable Energy Agency has indicated that such deep decarbonisation will require a transformation in the way we use energy: not only switching to largely renewable electricity sources, but also decarbonising industrial energy usages that are hard to electrify; for example high-grade heat, heavy freight, and chemical processing[2]. Hydrogen generated from renewable sources could be the key to realising this global energy transformation: by providing an energy-dense, zero-carbon fuel suitable for a range of industrial sectors ${ }^{3}$.

Hydrogen can be generated directly from the sun via electrolysis of water: sunlight provides the Gibbs free energy to drive the water splitting redox reaction (via the photovoltaic effect), resulting in oxygen and hydrogen. Many different stand-alone, photovoltaic-based hydrogen generators have been demonstrated on a lab scale. Solar cells can be electrically connected to standard electrochemical cells; 
commonly known as photovoltaic-electrolysis or PV-E ${ }^{45}$. Alternatively, photovoltaic components can be integrated with catalysts to create photoelectrodes; known as photoelectrochemical systems (PEC) ${ }^{67}$. A range of semiconductor materials and catalysts are under active investigation in the pursuit of highly efficient, low-cost, solar-to-hydrogen conversion ${ }^{89}$. While it is widely believed that PEC-type systems have the potential to be cheaper than PV-E ${ }^{10}$, is difficult to do detailed techno-economic comparisons of each technology, as nearly all of the proposed systems are in the research and development phase and reliable costings are not available.

The rapid rise of solar power over the last few decades provides important learning opportunities for renewable hydrogen generation. The global installed capacity of solar energy has increased exponentially since the early 2000 s, and photovoltaic installations accounted for over $20 \%$ of total growth in power generation in $2017^{11}$. Concurrently, economies of scale and increasing industrial experience have steadily reduced the price of silicon solar cells. The cost of silicon modules has now fallen to the point that they account for less than half of the total cost of an installed photovoltaic (PV) system, and are projected to fall further ${ }^{12}$. It follows that solar cell price is no longer a key driver for the PV industry, and instead increasing the solar cell efficiency is critical to continue to reduce the levelized cost of electricity (LCOE) from PV. A sustained effort to advance silicon solar cell technologies has increased the average efficiency of commercial PV modules by roughly $30 \%$ over the last 10 years ${ }^{13}$, while record Si solar efficiencies have climbed to $26.7 \%{ }^{14}$ - very close to the theoretically achievable efficiency limit. To continue driving down LOCE into the future, the PV community are looking to Sibased tandems as a viable option for increasing efficiencies past $30 \%{ }^{1516}$, and the International Technology Roadmap for PV predicts that Si tandem cells will be in mass production by $2020^{12}$. It is very likely that large-scale implementation of commercial PV-based solar hydrogen generators whether PV-E or PEC - will follow a similar learning curve as the PV industry, with concurrent cost reductions. Like PV systems, the balance of systems costs (i.e. all other installation costs) for solar hydrogen will scale with system area, and it has been argued that it is unlikely to be significantly lower for PEC than PV-E ${ }^{17}$. This suggest that the efficiency of solar-to-hydrogen conversion will be the key factor in reducing the levalised cost of hydrogen for any type of PV-based hydrogen generator. Rigorous techno economic analysis of different solar hydrogen pathways corroborates this, and identified system efficiency as the most important cost-reduction parameter ${ }^{18}$. Indeed, the U.S. Department of Energy has set a target solar-to-hydrogen efficiency of $20 \%$ by 2020 , to ensure PV-based renewable hydrogen is competitive in a future hydrogen economy ${ }^{19}$.

Like solar cells, the thermodynamic limits for PV-based solar-to-hydrogen (STH) conversion are determined by the bandgap of the semiconductor absorber. Assuming no reaction losses, the thermodynamic STH efficiency limit for hydrogen generators employing a single, optimum bandgap semiconductor is $30 \%$, close to that of PV cells. However, predictions taking into account unavoidable reaction overpotentials and realistic PV losses suggest that the practical limit to STH efficiency for systems based on a single junction PV component is much lower: between $10-15 \%{ }^{20,21}$. This is significantly lower than achievable PV efficiencies because water splitting requires a fixed voltage to 
drive the reaction, usually above $2.0 \mathrm{~V}$ for realistic electrochemical cells with typical overpotential losses. The band-gap of the semiconductor determines the photovoltage it can supply, and semiconductors with a sufficiently high bandgap to provide the required potential have a limited current output, as they can only use a small portion of the solar spectrum. One way to overcome this limit is to integrate two semiconductor absorbers with complementary bandgaps in a tandem configuration.

Theoretical studies investigating STH efficiencies of PV-based hydrogen systems have provided invaluable guidance in choosing complimentary semiconductor materials for tandem PV-based hydrogen systems ${ }^{20-25}$. Fountaine et al. have developed a cogent framework to calculate both limiting and realistic STH efficiencies, and the corresponding optimum bandgap configurations, introducing loss parameters to account for non-idealities in both the PV components and electrocatalysts ${ }^{21}$. This work predicted practical STH efficiency limits of $28 \%$ for tandem PEC systems based on high-performance, high-cost PV and catalysts materials, and $16 \%$ for low-cost materials. Other works have also considered the effect of system design on the limiting STH efficiencies ${ }^{22-24,26,27}$. In particular, Jacobsson et al. demonstrated that connecting several single-bandgap cells in series delivered the necessary photovoltage with comparable STH efficiencies, providing an economical alternative to tandems ${ }^{23}$. Despite the usefulness of the studies cited above, there have been few attempts to compare STH conversion limits for PV-E and PEC systems. Studies that do, assume a fixed electrolyser efficiency of between $68-73 \%$, and do not attempt a direct comparison of fundamental physical limitations of the different systems ${ }^{24,26}$. However, as Jacobsson and colleagues have eloquently argued, different types of PV-based hydrogen generators are essentially equivalent, as the fundamental physical processes in all systems are the same, regardless of the details of the system configuration ${ }^{28}$. While there are significant differences in the design requirements for different system types - for example whether or not the semiconductor needs to be able to withstand submersion in the electrolyte - all PV-based hydrogen generators must first convert optical energy into electrical energy via the photovoltaic effect, and then convert the electrical energy into chemical energy stored in hydrogen. This suggests that the performance of all types of PV-based solar hydrogen systems are limited by the same thermodynamic and physical considerations.

This article aims to provide guidance on how to rationally integrate PV components in solar hydrogen generators to optimise system performance, by providing a framework to compare theoretical STH efficiencies of different system configurations on equal footing. We calculate and compare the limiting and currently realisable efficiencies of a range of PV-based solar hydrogen systems, based on fundamental thermodynamic limitations, and experimentally demonstrated solar cell efficiencies and catalyst properties. We begin by considering the efficiency of the photovoltaic and electrochemical actions separately, and demonstrate the different ways they can be integrated in a PV-based solar hydrogen system using simple equivalent circuit models.

A key finding of this work is that if there is a direct interface between a PV component generating photovoltage and the catalyst, (as in PEC systems), then all the current must flow through that interface, and the PV component must be connected in series with the electrochemical components. This places 
restrictions on the way these components can be integrated into a solar hydrogen system, and specifically requires that the current generated by the photovoltaic action is directly coupled to the electrochemical action. Conversely, PV components with no direct PV-catalyst interfaces allow the inclusion of standard power management systems widely used in the solar PV industry, which can decouple the photovoltaic and electrochemical actions.

The results presented here clearly demonstrate that coupled systems will always have significantly lower efficiencies than the equivalent decoupled system when comparing identical components. Additionally, PEC-type coupled systems have much stricter material requirements to achieve the maximum possible STH efficiencies, and the bandgaps of the PV components will generally need to be optimised for a specific catalyst system and corresponding overpotential loss. Conversely, PV-E type decoupled systems provide better system performance for a given PV efficiency, and offer the opportunity to directly leverage advances in PV technologies, and the maturity of the solar industry, to rapidly advance renewable hydrogen generation.

\section{COUPLED, DECOUPLED AND HYBRID CONFIGURATIONS}

From a physical perspective, different routes to PV-based solar hydrogen generation are not fundamentally different, and instead exist on a continuum of device concepts ${ }^{28}$. At one end of the spectrum is PV-electrolysis, where solar cells are connected via wires to standard water splitting electrodes, as shown in Fig. 1(a, i). At the other extreme are wireless artificial leaf systems, where the semiconductors are directly integrated with the catalysts and the monolithic device is submerged in the electrolyte, shown in Fig.1(a,ii). In between are systems with one or more separate photoelectrodes, consisting of semiconductors integrated with catalysts, which can be connected via wires to each other or one or more solar cells. A representative configuration is shown in Fig.1(a,iii).

Most PV-based solar hydrogen generators combine two semiconductor components in a tandem configuration to provide the necessary photovoltage to drive the water splitting reaction. Tandems have been studied for solar photovoltaics for many decades, and it is illuminating to consider the different configurations that have been considered ${ }^{29}$. In 2-terminal configurations the two solar cells are connected in series, so that the photovoltages are summed, and the current in the system is limited by the cell with the lowest photocurrent. Alternately, solar cells can be connected in a 4-terminal configuration, where they are connected independently to a power management unit (PMU), and operated at their maximum power point. Power management can be provided by DC-DC convertors, which are widely used in the solar industry and are above $95 \%$ efficient ${ }^{30}$. The PMU can then sum the input power of both cells to provide a variable current and voltage output. There is also a variety of interesting work on three terminal connections ${ }^{31}$ and on sub modules ${ }^{32}$. The thermodynamic limiting efficiency is the same in all cases, but 4-terminal and sub-module connections offer a broader range of bandgap combinations that can achieve this limit ${ }^{32}$. 
(a) Coupled

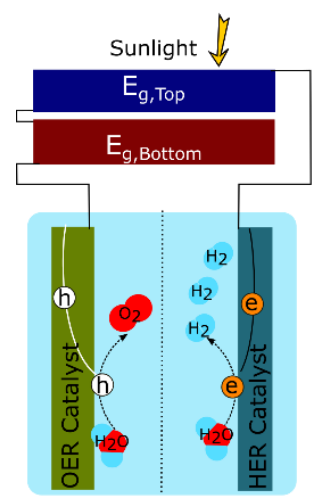

(i)

(d) Coupled

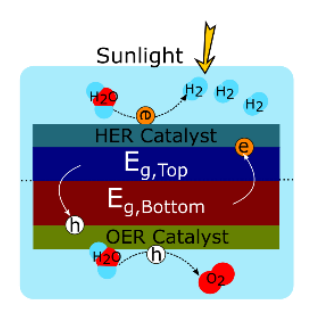

(ii)

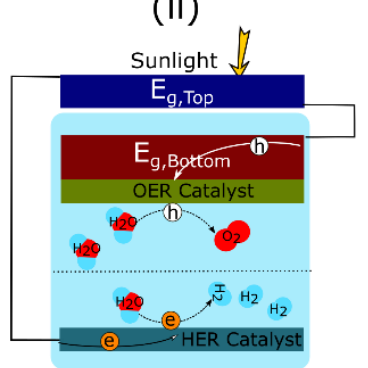

(iii)

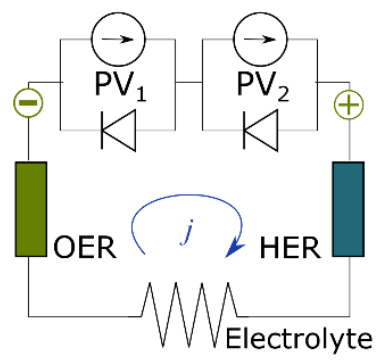

(b) Decoupled

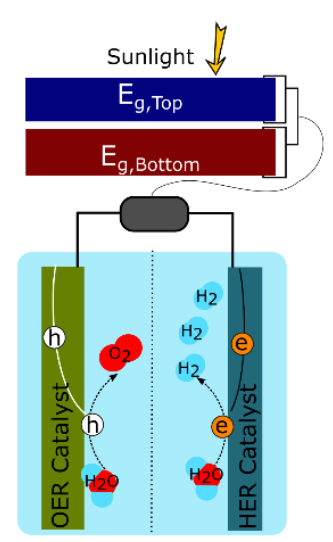

(e) Decoupled

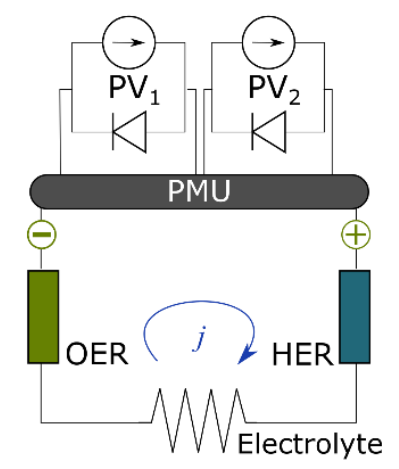

(c) Hybrid

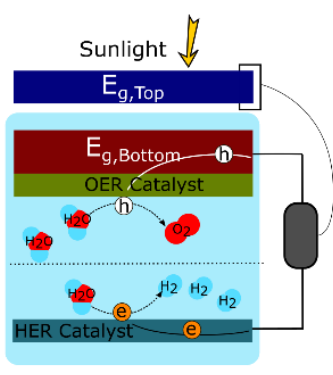

(f) Hybrid

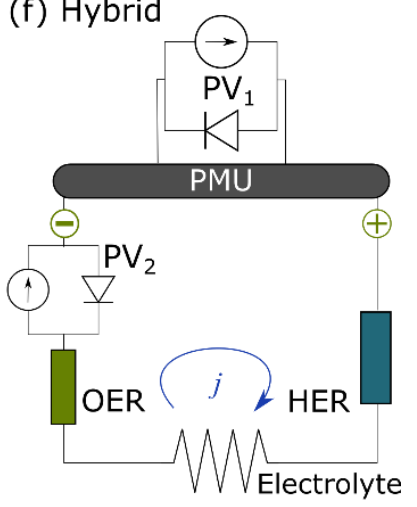

Fig.1 (a-c) Schematic of different PV-based solar hydrogen generators. In all cases two semiconductor photovoltaic (PV) components are used in a tandem configuration, with complementary bandgaps, where $E_{g_{-} T o p}>E_{g, \text { Bottom. }}$ The path of the electron, $e$, and hole, $h$, charge carriers is shown. HER is the hydrogen evolution reaction catalyst, OER is the oxygen evolution reaction catalyst, and PMU is a power management unit. (a) Schematic of different coupled configurations: (i) PV-electrolysis, (ii) artificial leaf, (iii) photoelectrocatalysis. (b) Schematic of a decoupled PV-electrolysis system. (c) Schematic of a hybrid system. (d-f) Equivalent circuit diagrams for (d) coupled, (e) decoupled, and (f) hybrid systems.

For the PV-based solar hydrogen systems shown in Fig.1(a), the PV components (either solar cells or photoelectrodes) and the electrochemical cell are connected in series with each other. We define these as coupled systems: the photovoltaic and electrochemical actions in the system are directly coupled since the same current must flow through all parts of the circuit. The three different configurations, shown schematically in Fig.1(a, i-iii), can all be represented by the same equivalent circuit diagram, given in Fig. 1(d): from an electrical point of view, the only difference is whether the electron and hole charge carriers are transported from one component to another through a wire or across an interface.

Previous studies that have investigated the limiting and realistic STH efficiencies of PV-based hydrogen systems for a range of semiconductor materials and catalysts ${ }^{20-24}$, have focused on coupled configurations that employ 2-terminal series connections between the PV components and the electrochemical cell, like those represented in Fig.1(a). However, by inspection of the schematics in Fig.1(a,ii), we can see that it is also possible to decouple the photovoltaic and electrochemical actions by 
introducing a power management unit (PMU), as shown schematically in Fig.1(b). In this configuration, represented by the equivalent circuit in Fig.1 (e), the elements of the electrochemical cell are still in series (catalysts and electrolyte), but the individual PV components are connected in a 4-terminal configuration. In this case the PV components can be operated at their maximum power points and the PMU provides the optimum output current and voltage required to drive the water splitting reaction. The current in each cell, and in the electrochemical cell, can now be optimised separately.

It is important to note that it is only possible to decouple a PV component if there is no direct integration of catalysts with the semiconductor. If catalysts are deposited on one of the PV components it must be in direct contact with the electrolyte. In this case all the generated current must flow through that interface, and hence the PV component must be connected in series with the electrochemical cell. Taking this into account, one can also imagine a hybrid system: where one of the PV components is connected via a PMU, while the second is a photoelectrode coupled to the electrochemical action, as shown in Fig.1(c). We represent this by the equivalent circuit in Fig. 1(f).

In the next section we introduce the efficiency models used in this work and demonstrate how the different coupling configurations affect the overall system efficiency.

\section{EFFICIENCY MODELS}

The photovoltaic effect occurs when the absorption of light in a material establishes a photocurrent and a photovoltage. In order to establish a photovoltage, photogenerated electrons and holes in a semiconductor must be separated and collected at different contacts. In other words, any semiconductor with charge carrier selective contacts can be considered a photovoltaic cell, whether the selectivity is due to a pn-junction, a heterojunction, hole/electron blocking layers, or chemically induced depletion regions ${ }^{33}$. It follows that the thermodynamic limiting efficiency for all photovoltaic

components is the same, and can be calculated using the Shockley-Quisser formulation ${ }^{34}$, regardless of its design or whether it is a solar cell, a photoelectrode, or a photoelectrochemcial cell.

For such an ideal PV component, all incident photons in the solar spectra with energies above the semiconductor bandgap energy, $E_{g}$, are absorbed and contribute to the photocurrent, $j_{L}$. The only loss that is considered is blackbody radiation from the cell at room temperature, in the form of the radiative recombination current density, $j_{\text {rad }}$. The current density dependent photovoltage of the cell is then given by:

$$
V_{P V}(j)=\frac{k_{B} T}{e} \ln \left(\frac{j_{L}-j}{j_{\text {rad }}}+1\right)
$$

where $k_{B}$ is the Boltzmann constant and $T$ is the temperature of the semiconductor. The power density provided by a PV cell is the product of the voltage and the current, and is maximised by operating the cell at the its maximum power point $M P P$. 
To drive electrochemical water splitting, the input voltage, $V_{I N}(j)$, must be large enough to overcome the voltage requirement of the reaction ( $\Delta E=1.23 \mathrm{~V}$ for water splitting), and any additional voltage losses introduced by the catalysts, $V_{O E R / H E R}(j)$ and by resistance to charge transport, $V_{e}(j)$. This can be written as:

$$
V_{I N}(j) \geq \Delta E+V_{O E R}(j)+V_{H E R}(j)+V_{e}(j)
$$

where $j$ is the current density through the electrochemical circuit. The power-to-hydrogen efficiency of an electrochemical cell is defined, relative to the input power, $P_{I N}$ by

$$
\eta_{P T H}=\frac{j \Delta E \eta_{F}}{P_{I N}}
$$

where $\eta_{F}$ is the Faradaic efficiency assumed to be unity in this work. In order to maximise the efficiency, it is necessary to find the optimum operating current density which maximises the current while minimising the current dependent voltage loss.

To model the full solar-to-hydrogen conversion efficiency, the photovoltage of the PV components, given by Eq.3, must be combined to provide the input voltage in Eq.7, and the input power, $P_{I N}$ in Eq.(10) is now the solar power density, $P_{A M 1.5}$. Inspection of the equivalent circuit models in Fig.1 determines how the different coupling configurations affect the overall system efficiency: the differences are solely due to the form of the input voltage.

Table 1: Form of the input voltage for different system configurations

\begin{tabular}{l|l} 
CONFIGURATION & FORM OF THE INPUT VOLTAG \\
\hline COUPLED & $V_{I N}(j)=V_{P V, 1}(j)+V_{P V, 2}(j)$ \\
DECOUPLED & $V_{I N}(j)=\frac{M P P_{1}+M P P_{2}}{j}$ \\
HYBRID & $V_{I N}(j)=\frac{M P P_{1}}{j}+V_{P V, 2}(j)$
\end{tabular}

In coupled configurations, all the components are in series and the input voltage must be provided by the sum of the current dependent photovoltages of both PV components, given in Eq.4. Conversely, decoupled systems allow the PV components to be connected in a 4-terminal configuration, meaning that the PV cells can be operated at their maximum power points. The in put voltage is then given by combining the output power of the solar cells and dividing by the operating current, given by Eq.5. In the hybrid case, one of the PV components is connected in series and the other is connected through a PMU. The input voltage is the sum of the current dependent photovoltage (Eq.6) of the first solar cell plus the voltage provided by the PMU. The equations for all three configurations are given in Table 1. 


\section{THERMODYNAMIC LIMITING EFFICICNCIES}

To investigate the effect of coupling or decoupling the system on the overall performance, we calculate the thermodynamic limiting STH efficiency as a function of the bandgap of the semiconductor PV components. The catalyst overpotenial is assumed to be negligible, i.e. $V_{O E R / H E R}=0 \mathrm{~V}$, as are resistance losses in the electrolyte, i.e. $V_{e}=0 \mathrm{~V}$, following the method of ref ${ }^{21}$. Note that these efficiencies are not achievable in practice, but provide an absolute upper limit for STH conversion.

Figure 2 shows the limiting STH efficiency, $\eta_{S T H}$, for (a) coupled, (b) decoupled, and (c-d) hybrid solar hydrogen systems, for different top and bottom bandgaps. It is informative to compare the coupled and hybrid STH efficiency with the efficiency of the corresponding PV-components connected in a 2-terminal configuration, $\eta_{2 T P V}$. The white contour lines indicate the system-to-PV efficiency ratio, $\frac{\eta_{S T H}}{\eta_{2 T P V}}$, as a percentage. Bandgap combinations that result in the maximum limiting efficiencies are summarised in Table 2 for different solar hydrogen configurations as well as 2-terminal tandem solar cells. A graph of the 2-terminal tandem solar cell efficiencies is given in Fig S1 of the supporting information.

The maximum STH efficiency for a coupled system is $40 \%$, indicated by a solid black circle in Fig.2(a), which agrees with previous work ${ }^{21}$. Even though we are neglecting reaction losses, this is significantly lower than the maximum efficiency of a 2-terminal tandem solar cell, and occurs for a different bandgap combination, as shown in Table 2. The differences between the STH and PV efficiencies are due to the fixed voltage requirements of the water splitting reaction. Unlike solar cells, which can be operated at optimum voltages and currents to maximise output power, the STH efficiency is determined by the fixed voltage requirements of the reaction and the variable operating current. Any additional voltage supplied by the power system above that required to overcome reaction losses will not be utilised in the system and will lead to additional power losses. This places stringent restrictions on bandgap combinations that can be employed to achieve high STH efficiency. The contour lines in Fig.2(a) show that that only a small fraction of the bandgap combinations achieve system-to-PV efficiency ratios above $90 \%$.

In contrast, decoupled systems are not restricted by any current or voltage matching requirements. As no reaction losses are included, the limiting STH efficiencies are identical to those of the corresponding 4-terminal tandem solar cells (i.e. $\frac{\eta_{\mathrm{STH}}}{\eta_{2 \mathrm{TPV}}}=100 \%$ for all bandgaps in Fig.2(b)), with a maximum of $46 \%$. Importantly, the limiting STH efficiency for decoupled systems is higher than that of coupled systems for all bandgap combinations. Indeed, Fig.2(b) shows that decoupled systems can exceed the maximum 

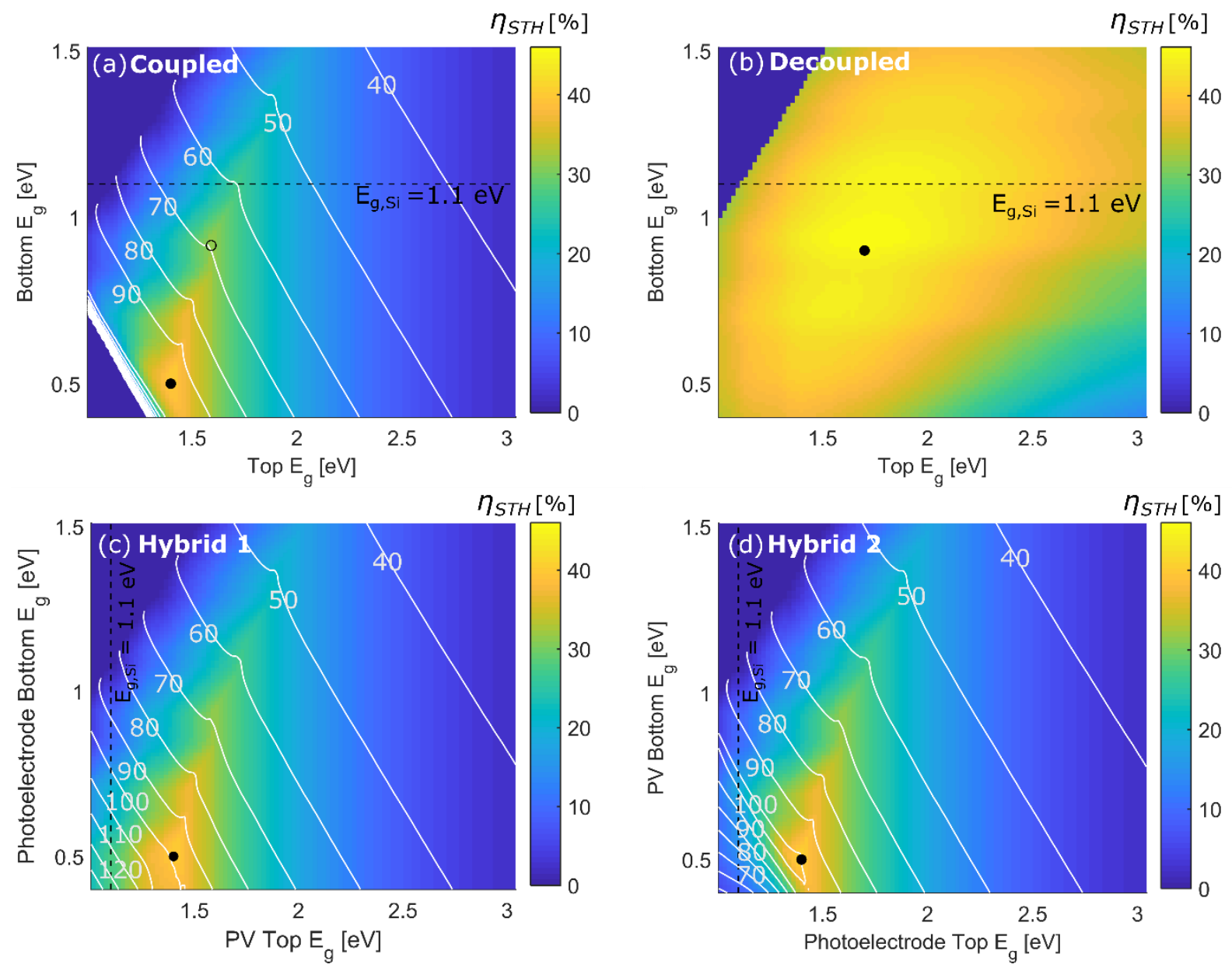

Figure 2. Calculated thermodynamic limiting STH efficiencies, $\eta_{S T H}$, for PV-based solar hydrogen systems in the following configurations: (a) Coupled, (b) Decoupled, (c) Hybrid 1 with the top PV component decoupled, and the bottom coupled (as shown in the schematic in Fig.1(c)), and (d) Hybrid 2 with the bottom PV component decoupled, and the top coupled. The white contour lines show the $\eta_{S T H}$ as a percentage of the corresponding 2-terminal tandem solar cell efficiency, $\frac{\eta_{S T H}}{\eta_{2 T P V}}$. The black closed dots indicate the bandgap combinations resulting in the optimum $\eta_{S T H}$ values for each configuration; the black open dot in Fig.2(a) represents the bandgap combination resulting in the optimum $\eta_{2 T P V}$.

efficiency of the coupled case with optimised tandem bandgaps, $\eta_{\mathrm{STH}}>40 \%$, over a broad range of bandgap combinations.

For hybrid systems, there are two possible configurations: hybrid 1 with a decoupled top bandgap solar cell incorporated with a coupled bottom bandgap photoelectrode (Fig. 2c); or hybrid 2 with a decoupled bottom bandgap solar cell connected to a coupled top bandgap photoelectrode (Fig. $2 \mathrm{~d}$ ). Both types of hybrid systems perform the same as a fully coupled configuration for almost all bandgap combinations. However, hybrid configurations show improved performance when both bandgaps are relatively small. Hybrid 1 systems also perform better than hybrid 2 systems at low bandgap combinations, and hybrid 1 systems can have system efficiencies larger than those of constituent 2-terminal PV tandem cells (i.e. 
$\frac{\eta_{\text {STH }}}{\eta_{\text {2TPV }}}>100$ ). This is because, like coupled systems, hybrid systems are limited by the photocurrent through the coupled cell, but the PMU can better utilise the power of the decoupled cell by providing the best current and voltage combination to match this current and minimise losses. Optimising the power output of the top cell (as in hybrid 1) provides a bigger performance boost than doing so for a bottom cell (in hybrid 2) as the top cell has access to a larger fraction of the solar spectrum, and hence has a higher efficiency. This means that

hybrid systems cannot exceed the maximum STH conversion limit of a coupled system at optimum bandgap configurations, where the cells are already providing sufficient voltage and the photocurrent is well matched, but can improve performance for small bandgaps as the PMU can increase the voltage output of the decoupled cell.

Given the commercial dominance of silicon solar cells and the rise of silicon tandems, it is useful to explicitly consider the case where one of the tandem PV components is silicon. Figure 3 shows limiting STH efficiencies for coupled, decoupled, and hybrid silicon PV-based solar hydrogen systems, as the bandap of the other PV component is varied. Note that for $E_{g}<1.1 \mathrm{eV}$ silicon is the top bandgap, while for $E_{g}>1.1 \mathrm{eV}$, silicon is the bottom bandgap. The STH efficiency for systems incorporating a single PV component is shown for comparison. Analogous to tandem systems, a single PV component can be connected to the electrochemical cell in series, resulting in a coupled system (Coupled 1J), or decoupled through a PMU (Decoupled 1J Si). Only PV bandgaps above $1.5 \mathrm{eV}$ have sufficient photovoltage to drive the reaction in an ideal coupled configuration, with a maximum thermodynamic limiting STH efficiency of $\eta_{S T H}=30 \%$, in agreement with previous results ${ }^{20,21}$. For the decoupled configuration, only an ideal single-junction silicon solar cell is considered.

Table 2 Summary of thermodynamic limiting efficiencies from data in Fig2

\begin{tabular}{l|cccc} 
IDEAL & COUPLED & DECOUPLED & HYBRID & 2-TERMINAL PV \\
\hline$\eta_{\max }$ & $40 \%$ & $46 \%$ & $40 \%$ & $46 \%$ \\
$E_{g, \text { top }}$ & $1.4 \mathrm{eV}$ & $1.7 \mathrm{eV}$ & $1.4 \mathrm{eV}$ & $1.6 \mathrm{eV}$ \\
$E_{g, \text { bottom }}$ & $0.5 \mathrm{eV}$ & $0.9 \mathrm{eV}$ & $0.5 \mathrm{eV}$ & $0.9 \mathrm{eV}$ \\
\hline SILICON BASED & COUPLED & DECOUPLED & HYBRID 1 & 2-TERMINAL PV \\
\hline$\eta_{\max }$ & $27 \%$ & $45 \%$ & $28 \%$ & $45 \%$ \\
$E_{g, \text { top }}$ & $1.7 \mathrm{eV}$ & $1.7-1.8 \mathrm{eV}$ & $1.1 \mathrm{eV}$ & $1.7 \mathrm{eV}$ \\
$E_{g, \text { bottom }}$ & $1.1 \mathrm{eV}$ & $1.1 \mathrm{eV}$ & $0.4 \mathrm{eV}$ & $1.1 \mathrm{eV}$
\end{tabular}

Figure 3 demonstrates that coupled silicon tandems have a thermodynamic STH efficiency limit of $\eta_{S T H}=27 \%$, lower than both decoupled single-junction silicon solar cells $\left(\eta_{S T H}=33 \%\right.$, ), and coupled single junction cells with ideal bandgaps. STH efficiency limits for the hybrid 1 configurations can only marginally exceed those of coupled systems for bottom bandgaps of less than $0.5 \mathrm{eV}\left(\eta_{S T H}=28 \%\right)$. In contrast, employing decoupled silicon tandems have the potential 
to significantly increase the STH conversion limits over the single junction case, with a maximum STH efficiency limit of $\eta_{S T H}=45 \%$.

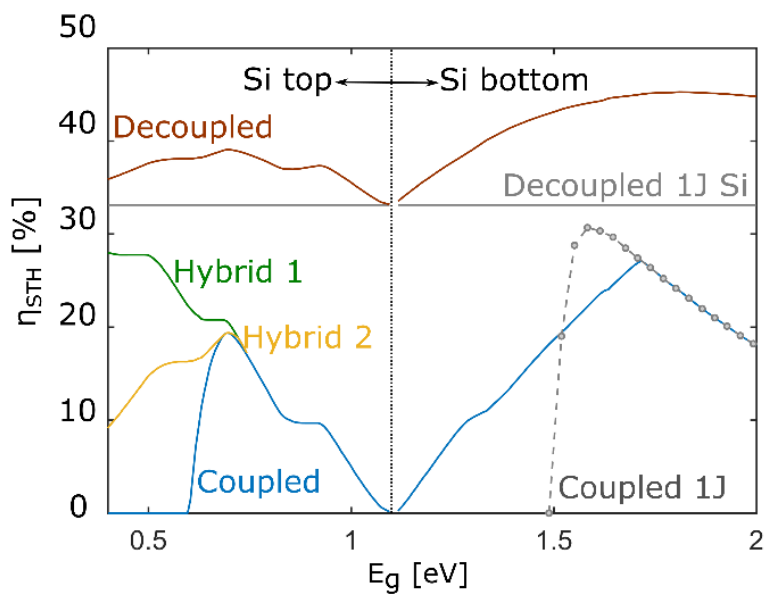

Figure 3. Calculated thermodynamic limiting STH efficiencies, $\eta_{S T H}$, for PV-based solar hydrogen systems with two PV components, where one of the PV components is silicon, with a bandgap $E_{g, S i}=1.1 \mathrm{eV}$. Data is shown for coupled and decoupled, and hybrid configurations. The efficiency of a PV-based solar hydrogen system driven by a single, decoupled, silicon PV component (Decoupled 1J Si) is also given, as is the STH effciency for a single, coupled PV component of varying bandgap energy (Coupled 1J).

\section{REALISTIC PV-BASED SOLAR HYDROGEN SYSTEMS}

Thermodynamic limits are useful to understand the potential of different system configurations, however they are unrealistic, as all electrochemical reactions suffer from reaction losses, which will affect the fixed voltage requirements. Additionally, all solar cells suffer from non-ideal diode characteristics, which will affect the photovoltage and photocurrent that they can supply. Given the strong dependence of STH efficiency on voltage losses for coupled systems due to current matching requirements, it is important to take into account non-idealities when comparing system configurations.

For this reason, we calculate the projected STH efficiencies for realistic systems. Again, we focus on silicon-based PV components due to their commercial relevance. We choose two different and technologically important silicon tandems with (i) a relatively high-cost, high-efficiency III-V top cell, and (ii) a potentially lost cost, and lower efficiency, Perovskite top cell. The III-V case is modelled on the work by Essig et al., who demostrated a $29.8 \%$ efficient, GaInP-Si tandem, measured in a 4-terminal configuration ${ }^{35}$. The Perovskite-Si tandem is based on recent work by Duong, who reported a $25.4 \%$ efficient 4-terminal tandem, using a rubidium multication Perovskite top cell with an optimsised bandgap ${ }^{36}$. Both cases represent important advancements in the field of tandem solar cells. 
We follow the approach of Fountaine and colleagues ${ }^{21,37}$ and use Butler-Volmer kinetics ${ }^{38}$ to model the over-potential of the catalysts employing realistic catalytic properties taken from literature. We choose the same catalyst parameters as ref ${ }^{21}$, consistent with data reported in refs ${ }^{3940}$ for high-cost catalysts like Pt and IrO2, and data from ref ${ }^{41}$ for low-cost catalysts like NiMo for HER, and NiZn, CoFe, and NiMoFe for OER, which generally have lower performance. We continue to neglect transport losses in the electrolyte $\left(V_{e}=0\right)$. Full details are provided in the supporting information.

The photovoltage of a realistic PV component is given by solving the standard trancendental solar cell equation, and introducing loss parameters to account for non-ideal behaviour. The non-ideal recombination current density is calculated as $j_{0}=\frac{j_{r a d}}{E R E}$, where the external radiative efficiency factor, $E R E$, quantifies the fraction of the total recombination that can be attributed to ideal radiative recombination. To determine non-ideal photocurrent we calculate realistic absorption spectra and define the collection efficiency, $f_{c}$, to account for any charge carrier collection losses. The absorption in the $\mathrm{Si}$ cell was calculated assuming Lambertian light trapping and no reflection loss, following the work of Green ${ }^{42}$, while a bandgap dependent analytical approximation was used to reproduce the shape of the absorption spectra of the top cells (details in the supporting information). An additional loss factor, $f_{\text {Para }}$, was defined for the top cells to account for parasitic absorption that would reduce the light incident on the bottom cell. Resistive losses due to carrier transport in the semiconductor are introduced through the series resistance term, $R_{S}$, and non-ideal diode characteristics are introduced through the shunt resistance, $R_{\text {sh }}$.

Table 3 Parameters for modelling realistic PV components

\begin{tabular}{l|ccc} 
PARAMETER & SI IBC & GAINP & PEROVSKITE \\
\hline External radiative efficiency factor, $E R E$ & $1.6 \mathrm{e}-3$ & $2.0 \mathrm{e}-1$ & $5.0 \mathrm{e}-6$ \\
Normalised series resistance, $\boldsymbol{r}_{\boldsymbol{s}}=\boldsymbol{R}_{\boldsymbol{s}} \frac{\boldsymbol{j}_{\boldsymbol{L}}}{\boldsymbol{V}_{\boldsymbol{o c}}}$ & $4.2 \mathrm{e}-3$ & $5.0 \mathrm{e}-2$ & $2.0 \mathrm{e}-1$ \\
Normalised shunt resistance, $\boldsymbol{r}_{\boldsymbol{s} \boldsymbol{h}}=\boldsymbol{R}_{\boldsymbol{s} \boldsymbol{h}} \frac{\boldsymbol{j}_{\boldsymbol{L}}}{\boldsymbol{V}_{\boldsymbol{o c}}}$ & $1.8 \mathrm{e} 5$ & $1.0 \mathrm{e} 3$ & $1.0 \mathrm{e} 2$ \\
Collection efficiency, $\boldsymbol{f}_{\boldsymbol{c}}$ & 0.94 & 0.70 & 0.85 \\
Parasitic absorption factor, $\boldsymbol{f}_{\boldsymbol{P a r} \boldsymbol{a}}$ & NA & 0.15 & 0.10
\end{tabular}

The loss parameters were fitted to reproduce the reported experimental current-voltage curves and solar cell figures of merit in refs ${ }^{3536}$. Where possible, the parameters for the silicon bottom cell were taken directly from measurements of an integrated back contact (IBC) cell reported by Franklin ${ }^{43}$, as this is similar to the bottom cell used in the Perovskite-Si tandem modelled in this work ${ }^{36}$. The experimentally measured recombination current (i.e. the reverse-biased dark current from ref ${ }^{43}$ ) was used to to calculate $E R E$ for the silicon cell. Since both GaInP and Perovskite offer the possibility of bandgap tuning to optimise STH efficiency, we calculate the STH efficiency for a range of top cell bandgaps. A detailed description of the realistic solar cell modelling and fitting procedure, as well as the calculated IV curves, absorption spectra, and FoM, are included in the SI. The model inputs, including fitted loss parameters, are summarised in Table 3. 
Figure 4 (a-b) shows calculated STH efficiencies for realistic silicon tandem-based hydrogen generators incorporating (a) Perovskite-Si and (b) GalnP-Si tandems with varying top bandgaps. Data is shown for coupled and decoupled solar hydrogen configurations, as well as a hybrid 1 system with a coupled bottom silicon photoelectrode and a decoupled top solar cell with a varying bandgap. For comparison, we show STH efficiencies for single-junction solar cells of varying bandgap in coupled and decoupled configurations in Fig.4(c), modelled with the same bandgap dependent loss parameters as the silicon bottom cell (given in Table 3). The shaded areas on both graphs represent the variation in the STH efficiencies when using different catalysts: the upper bound is the limit for high efficiency, high-cost catalysts, while the lower bound is the limit for low-cost catalysts with lower performance. The solar cell efficiencies for the modelled PV components are given in the corresponding graphs and the square markers show the experimental efficiency measured for the solar cells in refs ${ }^{433536}$.
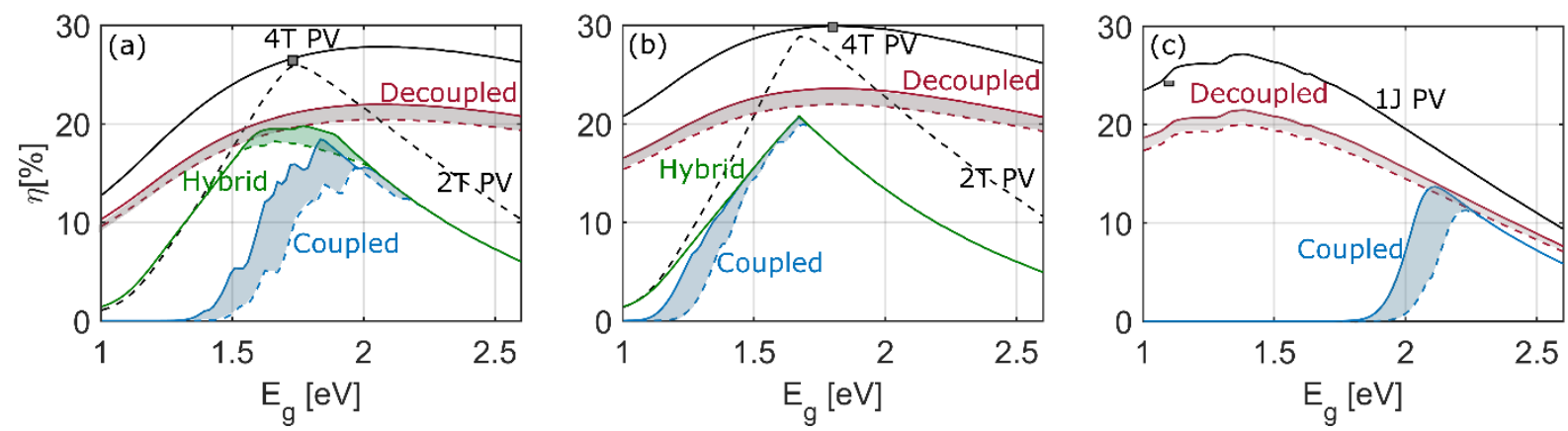

Figure 4. Calculated STH efficiencies for solar hydrogen generators based on realistic (a) Perovskite-Si tandems and (b) GaInP-Si tandems with varying top bandgaps. Data is shown for systems in coupled, decoupled, and hybrid configuration. Similar results for are given for a coupled and decoupled single-junction solar cell of varying bandgap (c), modelled with the same loss parameters as the Si bottom cell. The shaded areas show effect of catalysts: the upper bound (solid line) for high cost catalysts, the lower bound (dashed line) for low cost catalysts.

Figure 4 demonstrates that decoupled systems achieve much higher STH efficiencies with much less stringent bandgap requirements than the hybrid or coupled configurations for both the tandems and the single junction solar cells. Additionally, all three decoupled systems can exceed the DOE target STH efficiency target for 2020 of $20 \%$, with both low- and high-cost catalysts. Coupled systems employing high efficiency GalnP-Si tandems can only reach $20 \%$ when the top bandgap is optimised at $E_{g}=$ $1.67 \mathrm{eV}$.

It is also notable that the relationship between STH and PV efficiency for coupled systems changes significantly for different combinations of PV components and catalysts. Perovskite top cells suffer from more severe recombination (smaller ERE) and non-ideal diode characteristics (lower $R_{\text {sh }}$ ) than GalnP top cells. This means that Perovskite cells need a larger bandgap to provide the photovoltage necessary to drive the reaction, pushing the maximum STH efficiency to higher bandgaps where the PV efficiency is lower. This is similar to the single junction case in Fig.4(c), where a very large bandgap is required to 
overcome the voltage requirements of the reaction and catalyst losses, far from the optimal for power conversion efficiency, limiting practical STH efficiencies to $11-14 \%$ depending on the catalysts used, in agreement with previous calculations ${ }^{20,21}$. Changing the catalyst from high to low performance increases the photovoltage required, again shifting the optimal bandgap to higher energies for both Perovskite and single junction cells.

Conversely, the GalnP top cell is able to provide enough photovoltage to drive the reaction and compensate for catalytic losses at the bandgap corresponding to the maximum PV efficiency. As a result, the maximum STH and PV efficiency occur at the same optimal bandgap for the coupled case. Increasing the reaction losses by switching to a low-cost catalyst results in only a small performance reduction.

The realistic calculations show that the optimal bandgap of the Perovskite PV component in a coupled solar hydrogen system would be different from that used in a tandem solar cell, and would need to be optimised independently for different catalysts. For example, Fig.4(a) demonstrates that integrating a Perovskite-Si tandem cell designed for maximum 2-terminal PV performance ( $E_{g}=1.73 \mathrm{eV}$ ) into a coupled system would result in STH efficiencies of roughly $15 \%$ and $35 \%$ less than the maximum possible if the bandgap were optimised for water splitting with high- and low-cost catalysts respectively $\left(E_{g}=\right.$ $1.84 \mathrm{eV}, E_{g}=1.97 \mathrm{eV}$ ). This is likely to be generally true for other top-cell materials that have low radiative efficiency and shunt resistances.

In the hybrid configuration, decoupling the Perovskite top cell largely compensates for the non-ideal PV characteristics and reduces the effect of the catalyst performance on the maximum STH efficiency, as the PMU can simply increase the voltage. This results in higher overall STH efficiencies for both types of catalysts, and a similar optimal bandgap for both STH and PV maximum efficiency. For the GalnP top cell, the differences between high-cost and low-cost catalysts are completely eliminated by decoupling the top cell. Interestingly, using high-cost catalysts has no effect on the maximum STH efficiency as it is limited by the photocurrent through the bottom coupled cell, not the voltage.

Fully decoupled systems exhibit a much more straightforward relationship between STH and PV efficiency. Differences in the non-ideal characteristics of the cells have a negligible effect on the shape of the STH efficiency curve as the PMU can modulate either the voltage or current of either cell to compensate. Additionally, reducing the catalyst performance by going from high to low cost materials lowers STH efficiency in a predictable manner, largely independent of the top cell bandgap. This means that decoupled solar hydrogen system systems allow advances in high efficiency tandem solar cells to be directly employed for solar hydrogen generation.

The calculations presented here have assumed standard "1-sun" solar illumination; however solar hydrogen systems will need to operate under a range of illumination conditions throughout the diurnal cycle and it is worth noting the effect of this variation. Calculations presented in Figure S3 of the Supporting Information demonstrate that intensity only weakly effects the STH efficiency and the conclusions presented above hold when the input irradiance varies from 0.5 to 1.5 times the standard (AM1.5g) solar spectra. 


\section{SUMMARY AND CONCLUSIONS}

Despite the fact that the fundamental physical processes are the same in different stand-alone, PVbased solar hydrogen systems, system configuration has a big impact on both thermodynamic limiting efficiencies and predicted realistic efficiencies.

The calculations presented in this work show that decoupled solar hydrogen systems have the potential to be significantly more efficient than coupled systems employing identical PV materials and catalysts. Importantly, decoupled systems with single-junction silicon solar cells and high cost catalysts exhibit achievable STH efficiencies above $20 \%$, meeting the DOE target efficiency for $2020^{19}$. Additionally, the realistic Perovskite-Si tandems, and GalnP-Si tandems considered here are all predicted to exceed STH efficiencies of over $20 \%$ in conjunction with low-cost catalysts in a decoupled configuration. This demonstrates that PV-E-type, decoupled stand-alone systems could directly leverage silicon PV technologies that have been developed for the solar power industry to rapidly advance solar hydrogen generation.

While it is possible to meet the $20 \%$ STH efficiency target with coupled system configurations, the bandgap requirements for the PV components are much more stringent, and may need to be optimised for a given catalyst for materials with low radiative efficiency. This will likely make it more challenging to incorporate existing PV technologies into PEC-type coupled solar hydrogen systems.

We have purposely avoided a techno-economic comparison of the systems considered in this article due to the difficulty in determining accurate costs for emerging technologies, however it is possible to drawn some very general conclusions from the result presented here. Even with aggressive cost reductions in high efficiency tandem PV components, it is very unlikely that they will ever be cheaper than single junction silicon cells. It follows that system configurations employing high efficiency tandems should only be considered if they demonstrate the potential for significantly higher STH efficiency than systems employing a single junction silicon solar cell. The results presented above suggest that this is only likely to occur in PV-E-type decoupled configurations. Conversely, systems employing PEC-type coupled PV components will likely have to employ novel device concepts that are significantly cheaper, and require lower balance of systems costs, than standard PV solar cells. This aligns with the technoeconomic analysis of Shaner and colleagues, who concluded that for hydrogen PEC systems to be cost competitive, radically new ideas and materials will be needed, with the potential for low-cost and large-scale implementation ${ }^{18}$. As pointed out in a recent Opinion from Jacobsson ${ }^{17}$, this may prove to be an insurmountable task.

\section{AKNOWLEDGMENTS}

The author would like to thank Dr Matthew Stocks and Prof Kylie Catchpole for invaluable discussions, and Prof Andrew Blakers for the conversation that sparked off the line of inquiry. This work is funded by 
the Australian Renewable Energy Agency (grant number KC007). Dr Beck is the recipient of an Australian Research Council Discovery Early Career Award (project number DE180100383) funded by the Australian Government.

\section{SUPPORTING INFORMATION}

- Details of the solar-to-hydrogen efficiency models including the fitting procedure for the realistic systems

- Thermodynamic limiting efficiencies for tandem solar cells in a 2-terminal configuration

- Calculated IV and absorption spectra for GaInP-Si tandems and Perovskite-Si tandems modelled in this work

- Effect of illumination intensity on the solar-to-hydrogen efficiency and output current of realistic solar hydrogen generators employing Perovskite-Si tandems modelled in this work

\section{TOC GRAPHIC}

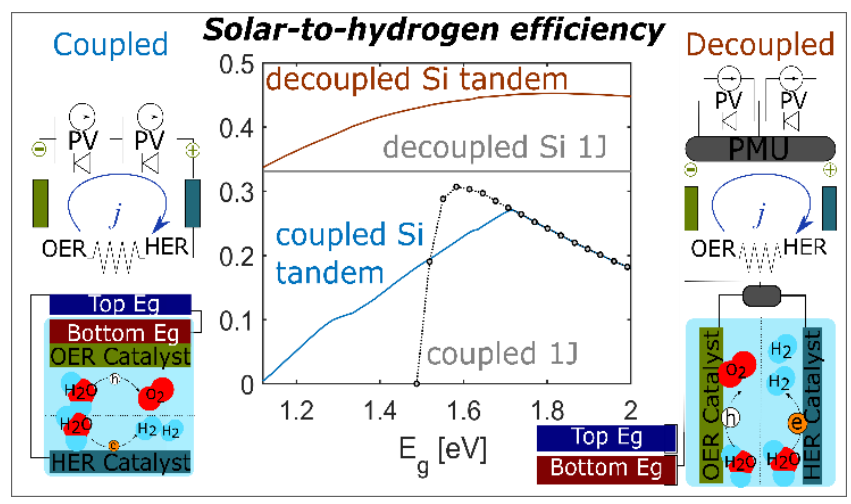

\section{REFERENCES:}

(1) V. Masson-Delmotte; Zhai, P.; Pörtner, H. O.; Roberts, D.; Skea, J.; Shukla, P. R.; Pirani, A.; Moufouma-Okia, W.; Péan, C.; Pidcock, R.; Connors, S.; Matthews J. B. R.; Chen, Y.; Zhou, X.; Gomis, M. I.; Lonnoy, E.; Maycock, T.; Tignor, M.; Waterfield, T. IPCC, 2018: Summary for Policymakers. In: Global Warming of $1.5^{\circ} \mathrm{C}$. An IPCC Special Report on the Impacts of Global Warming of $1.5^{\circ} \mathrm{C}$ above Pre-Industrial Levels and Related Global Greenhouse Gas Emission Pathways, in the Context of Strengthening the Global; Geneva, Switzerland, 2018.

(2) IRENA. Global Energy Transformation - A Roadmap to 2050; Abu Dhabi, 2018.

(3) IRENA. Hydrogen from Renewable Power: Technology Outlook for the Energy Transition; Abu Dhabi, 2018.

(4) Jia, J.; Seitz, L. C.; Benck, J. D.; Huo, Y.; Chen, Y.; Ng, J. W. D.; Bilir, T.; Harris, J. S.; Jaramillo, T. F. Solar Water Splitting by Photovoltaic-Electrolysis with a Solar-to-Hydrogen Efficiency over $30 \%$. Nat. Commun. 2016, 77 (23), 13237. https://doi.org/10.1038/ncomms13237. 
(5) Bonke, S. A.; Wiechen, M.; MacFarlane, D. R.; Spiccia, L. Renewable Fuels from Concentrated Solar Power: Towards Practical Artificial Photosynthesis. Energy Environ. Sci. 2015, 8 (9), 27912796. https://doi.org/10.1039/C5EE02214B.

(6) Young, J. L.; Steiner, M. A.; Döscher, H.; France, R. M.; Turner, J. A.; Deutsch, T. G. Direct Solar-toHydrogen Conversion via Inverted Metamorphic Multi-Junction Semiconductor Architectures. Nat. Energy 2017, 2 (4), 1-8. https://doi.org/10.1038/nenergy.2017.28.

(7) Verlage, E.; Hu, S.; Liu, R.; Jones, R. J. R.; Sun, K.; Xiang, C.; Lewis, N. S.; Atwater, H. A. A Monolithically Integrated, Intrinsically Safe, $10 \%$ Efficient, Solar-Driven Water-Splitting System Based on Active, Stable Earth-Abundant Electrocatalysts in Conjunction with Tandem III-V Light Absorbers Protected by Amorphous TiO 2 Films † Broader Context. Energy Environ. Sci 2015, 8, 3166. https://doi.org/10.1039/c5ee01786f.

(8) Roger, I.; Shipman, M. A.; Symes, M. D. Earth-Abundant Catalysts for Electrochemical and Photoelectrochemical Water Splitting. Nat. Rev. Chem. 2017, 1. https://doi.org/10.1038/s41570016-0003.

(9) Montoya, J. H.; Seitz, L. C.; Chakthranont, P., Vojvodic, A.; Jaramillo, T. F., Norskov, J. K. Materials for Solar Fuels and Chemicals. Nature Materials. 2016, 16 (1), 70-81. https://doi.org/10.1038/nmat4778.

(10) Chu, S.; Li, W.; Yan, Y.; Hamann, T.; Shih, I.; Wang, D.; Mi, Z. Roadmap on Solar Water Splitting: Current Status and Future Prospects. Nano Futur. 2017, 1 (2), 022001. https://doi.org/10.1088/2399-1984/aa88a1.

(11) BP. Statistical Review of World Energy 2018. 2018, 1-53.

(12) International Technology Roadmap for Photovoltaic (ITRPV) Results, Ninth Edition; 2018. https://doi.org/http://www.itrs.net/Links/2013ITRS/2013Chapters/2013Litho.pdf.

(13) Fraunhofer ISE. PHOTOVOLTAICS REPORT: Presented by Fraunhofer Institute for Solar Energy Systems, ISE with Support of PSE Conferences \& Consulting GmbH; Freiburg, 2018.

(14) Green, M. A.; Hishikawa, Y.; Dunlop, E. D.; Levi, D. H.; Hohl-Ebinger, J.; Ho-Baillie, A. W. Y. Solar Cell Efficiency Tables (Version 51). Prog. Photovoltaics Res. Appl. 2018, 26 (1), 3-12. https://doi.org/10.1002/pip.2978.

(15) Yu, Z.; Leilaeioun, M.; Holman, Z. Selecting Tandem Partners for Silicon Solar Cells. Nat. Energy 2016, 1 (11). https://doi.org/10.1038/nenergy.2016.137.

(16) White, T. P.; Lal, N. N.; Catchpole, K. R. Tandem Solar Cells Based on High-Efficiency for $>30 \%$ Efficiency. IEEE J. Photovoltaics 2014, 4 (1), 208-214. https://doi.org/10.1109/JPHOTOV.2013.2283342.

(17) Jacobsson, T. J. Photoelectrochemical Water Splitting: An Idea Heading towards Obsolescence? 
2018. https://doi.org/10.1039/c8ee00772a.

(18) Shaner, M. R.; Atwater, H. A.; Lewis, N. S.; McFarland, E. W. A Comparative Technoeconomic Analysis of Renewable Hydrogen Production Using Solar Energy. Energy Environ. Sci. 2016, 9 (7), 2354-2371. https://doi.org/10.1039/c5ee02573g.

(19) U.S. Department of Energy (DOE). DOE Technical Targets for Hydrogen Production from Photoelectrochemical Water Splitting https://www.energy.gov/eere/fuelcells/doe-technicaltargets-hydrogen-production-photoelectrochemical-water-splitting (accessed Jan 23, 2018).

(20) Bolton, J. R.; Strickler, S. J.; Connolly, J. S. Limiting and Realizable Efficiencies of Solar Photolysis of Water. Nature 1985, 316 (6028), 495-500. https://doi.org/10.1038/316495a0.

(21) Fountaine, K. T.; Lewerenz, H. J.; Atwater, H. A. Efficiency Limits for Photoelectrochemical WaterSplitting. Nat. Commun. 2016, 7, 1-9. https://doi.org/10.1038/ncomms13706.

(22) Kemppainen, E.; Halme, J.; Lund, P. Physical Modeling of Photoelectrochemical Hydrogen Production Devices. J. Phys. Chem. C 2015, 119 (38), 21747-21766.

https://doi.org/10.1021/acs.jpcc.5b04764.

(23) Jacobsson, T. J.; Fjällström, V.; Edoff, M.; Edvinsson, T. A Theoretical Analysis of Optical Absorption Limits and Performance of Tandem Devices and Series Interconnected Architectures for Solar Hydrogen Production. 2015. https://doi.org/10.1016/j.solmat.2015.02.023.

(24) Hu, S.; Xiang, C.; Haussener, S.; Berger, A. D.; Lewis, N. S. An Analysis of the Optimal Band Gaps of Light Absorbers in Integrated Tandem Photoelectrochemical Water-Splitting Systems. Energy Environ. Sci. 2013, 6 (10), 2984. https://doi.org/10.1039/c3ee40453f.

(25) Ross, R. T. Some Thermodynamics of Photochemical Systems. J. Chem. Phys. 1967, 46 (12), 45904593. https://doi.org/10.1063/1.1840606.

(26) Rothschild, A.; Dotan, H. Beating the Efficiency of Photovoltaics-Powered Electrolysis with Tandem Cell Photoelectrolysis. ACS Energy Lett. 2017, 2 (1), 45-51. https://doi.org/10.1021/acsenergylett.6b00610.

(27) Segev, G.; Beeman, J. W.; Greenblatt, J. B.; Sharp, I. D. Hybrid Photoelectrochemical and Photovoltaic Cells for Simultaneous Production of Chemical Fuels and Electrical Power. Nat. Mater. 2018, 17 (12), 1115-1121. https://doi.org/10.1038/s41563-018-0198-y.

(28) Jacobsson, T. J.; Fjällström, V.; Edoff, M.; Edvinsson, T. Sustainable Solar Hydrogen Production: From Photoelectrochemical Cells to PV-Electrolyzers and Back Again. Energy Environ. Sci. 2014, 7 (7), 2056. https://doi.org/10.1039/c4ee00754a.

(29) Gee, J. M. A Comparison of Different Module Configurations for Multi-Band-Gap Solar Cells. Sol. Cells 1988, 24 (1-2), 147-155. https://doi.org/10.1016/0379-6787(88)90044-0. 
(30) Enrique, J. M.; Durán, E.; Sidrach-de-Cardona, M.; Andújar, J. M. Theoretical Assessment of the Maximum Power Point Tracking Efficiency of Photovoltaic Facilities with Different Converter Topologies. Sol. Energy 2007, 81 (1), 31-38. https://doi.org/10.1016/J.SOLENER.2006.06.006.

(31) Nagashima, T.; Okumura, K.; Murala, K.; Kimura, Y. Three-Terminal Tandem Solar Cells with a Back-Contact Type Bottom Cell. Conf. Rec. IEEE Photovolt. Spec. Conf. 2000, 2000-Janua, 11931196. https://doi.org/10.1109/PVSC.2000.916102.

(32) Stocks, M.; Loo, Y. X.; Lal, N. N. Free The Bandgap! Series-Parallel Connection Of Tandem Cells. In 32nd European Photovoltaic Solar Energy Conference and Exhibition; Munich, 2016; Vol. 1BV.6.12, pp 223-227. https://doi.org/10.4229/EUPVSEC20162016-1BV.6.12.

(33) Wurfel, U.; Cuevas, A.; Wurfel, P.; Würfel, U. Charge Carrier Separation in Solar Cells. IEEE J. Photovoltaics 2015, 5 (1), 461-469. https://doi.org/10.1109/JPHOTOV.2014.2363550.

(34) Shockley, W.; Queisser, H. J. Detailed Balance Limit of Efficiecny of P-n Junction Solar Cells. J. Appl. Phys. 1961, 32 (3), 510.

(35) Essig, S.; Steiner, M. A.; Allebé, C.; Geisz, J. F.; Paviet-Salomon, B.; Ward, S.; Descoeudres, A.; LaSalvia, V.; Barraud, L.; Badel, N.; Faes, A.; Levrat, J.; Despeisse, M.; Ballif, C.; Stradins, P.; Young, D. L. Realization of GaInP/Si Dual-Junction Solar Cells with 29.8\% 1-Sun Efficiency. IEEE J. Photovoltaics 2016, 6 (4), 1012-1019. https://doi.org/10.1109/JPHOTOV.2016.2549746.

(36) Duong, T.; Wu, Y. L.; Shen, H.; Peng, J.; Fu, X.; Jacobs, D.; Wang, E. C.; Kho, T. C.; Fong, K. C.; Stocks, M. Franklin, E.; Blakers, A.; Zin, N., Mclntosh, K.; Li, W.; White, T. P.; Weber, K.; Catchpole, K. R. . Rubidium Multication Perovskite with Optimized Bandgap for Perovskite-Silicon Tandem with over 26\% Efficiency. Adv. Energy Mater. 2017, 7 (14), 1-11.

https://doi.org/10.1002/aenm.201700228.

(37) Shaner, M. R.; Fountaine, K. T.; Lewerenz, H. J. Current-Voltage Characteristics of Coupled Photodiode-Electrocatalyst Devices. Appl. Phys. Lett. 2013, 103 (14).

https://doi.org/10.1063/1.4822179.

(38) Bard, A. J.; Faulkner, L. R. ELECTROCHEMICAL METHODS Fundamentals and Applications, 2nd ed.; John Wiley and Sons, Inc.: New York, 1944. https://doi.org/10.1016/B978-0-12-381373-2.000569.

(39) Trasatti, S. Work Function, Electronegativity, and Electrochemical Behaviour of Metals: III. Electrolytic Hydrogen Evolution in Acid Solutions. J. Electroanal. Chem. Interfacial Electrochem. 1972, 39 (1), 163-184. https://doi.org/10.1016/S0022-0728(72)80485-6.

(40) Blouin, M.; Guay, D. Activation of Ruthenium Oxide, Iridium Oxide, and Mixed Ru[Sub x]Ir[Sub 1-x] Oxide Electrodes during Cathodic Polarization and Hydrogen Evolution. J. Electrochem. Soc. 1997, 144 (2), 573. https://doi.org/10.1149/1.1837450. 
(41) McCrory, C. C. L.; Jung, S.; Ferrer, I. M.; Chatman, S. M.; Peters, J. C.; Jaramillo, T. F. Benchmarking Hydrogen Evolving Reaction and Oxygen Evolving Reaction Electrocatalysts for Solar Water Splitting Devices. J. Am. Chem. Soc. 2015, 137 (13), 4347-4357.

https://doi.org/10.1021/ja510442p.

(42) Green, M. A. Lambertian Light Trapping in Textured Solar Cells and Light-Emitting Diodes: Analytical Solutions. Prog. Photovoltaics Res. Appl. 2002, 10 (4), 235-241.

https://doi.org/10.1002/pip.404.

(43) Franklin, E.; Fong, K.; McIntosh, K.; Fell, A.; Blakers, A.; Kho, T.; Walter, D.; Wang, D.; Zin, N.; Stocks, M.; Wang, E.; Grant, N.; Wan, Y.; Yang, Y.; Zhang, X.; Feng, Z.; Verlinden, P. J. Design, Fabrication and Characterisation of a $24.4 \%$ Efficient Interdigitated Back Contact Solar Cell. Prog. Photovoltaics Res. Appl. 2016, 24 (4), 411-427. https://doi.org/10.1002/pip.2556. 